\section{Rehabilitation management of Pompe disease, from childhood trough adulthood: A systematic review of the literature}

\author{
Bruno Corrado, Gianluca Ciardi, \\ Clemente Servodio Iammarrone \\ Department of Public Health, 'Federico \\ II' University of Naples, Italy
}

\section{Abstract}

Pompe disease (PD) is a rare neuromuscular disorder caused by a deficiency of the enzyme acid alpha-glucosidase. There are three forms of PD depending on the age at onset and clinical severity. PD causes involvement of different organ systems, such as the heart, musculoskeletal system, and respiratory system. As of today, enzyme replacement therapy represents the main therapeutic tool for PD. Rehabilitation is an integral part of a multidisciplinary approach to this pathology. The goal of the present review is to find scientific evidence for the rehabilitative approach to $\mathrm{PD}$, with respect to both the infantile- and adult-onset forms. A systematic literature review was made using the following databases: Pubmed, Pedro, Cochrane Library, EDS Base Index, Trip, and Cinhal. Randomized controlled trials or cohort studies with a sample population of at least six subjects were retrieved. The PICO method was used to formulate the clinical query. The search resulted in 1665 articles. Of these, four cohort studies were subjected to the final phase of the review. Three studies regarded inspiratory muscle training with a threshold, while the fourth study analyzed the effectiveness of therapeutic, aerobic, and reinforcement exercises. Inspiratory muscle training with a threshold increases the pressures generated during inhalation. Aerobic exercise is capable of increasing patients' muscular endurance and performance. To date, however, rehabilitative treatment for patients with $\mathrm{PD}$ has no validation in evidencebased medicine. Further studies, possibly with a larger sample size and higher quality are necessary to confirm the effectiveness of rehabilitation in patients with $\mathrm{PD}$.

\section{Introduction}

Pompe disease (PD), also referred to as acid maltase deficiency (AMD) or glycogen storage disease type II (GSDII), is a rare autosomal recessive neuromuscular disorder caused by a deficiency of the lysosomal enzyme acid alpha-glucosidase (GAA). ${ }^{1}$ A shortage of GAA causes a toxic accumulation of glycogen in many tissues, and in particular in smooth, skeletal, and cardiac muscle. The incidence of the syndrome is variable and depends upon ethnicity and geographical area. It is more prevalent among African-Americans $(1: 14000)^{2}$ and much less on the European continent $(1: 40000))^{3}$ From the etiological perspective, PD is caused by different mutations of the genes that interact to synthesize GAA, with a consequent lysosomal storage of glycogen. The mutations most studied in the literature are c. $525 \mathrm{delT},{ }^{4}$ c. $2237 \mathrm{G}>\mathrm{A},{ }^{5}$ and c. $-32-13 \mathrm{~T}>$ G. ${ }^{6}$ An increasing number of studies have dealt with the role of autophagy in the genesis of PD. Autophagy, or 'self-eating', is an extremely complex lysosomal degradation process controlled by a network of multiple genes. The main function of this mechanism is to eliminate organelles and obsolete protein aggregates in order to protect the cell from the stress of oxidation and/or nutritional deficiency. Changes in this mechanism are linked to various neurodegenerative pathologies involving accumulation, such as PD, and in the generation of some forms of tumors with cellular senescence. 7-10

PD is classified into two forms according to the age of onset. The infantile form (infantile-onset Pompe disease - IOPD) clinically arises in the first year of life, while the adult form (late-onset Pompe disease - LOPD) appears between 29 and 33 years of age. ${ }^{11}$ IOPD in turn is classified into two types depending on organ involvement and degree of myopathy: the classical form (classic IOPD), which usually leads to death due to cardiac arrest within the first year of life, and the non-classical form (non-classic IOPD), which is characterized by cardiomegaly and muscular weakness, with consequent respiratory insufficiency. Patients affected by non-classic IOPD are not faced with death due to cardiac arrest within the first year of life but generally do not survive beyond early childhood due to serious respiratory problems.

PD damages different organ systems in the body. With respect to the respiratory system, patients develop progressive respiratory insufficiency due to weakness of the diaphragm and intercostal muscles. Effects on the musculoskeletal system include insufficiency of the pelvic girdle muscles, insufficiency of the neck flexor muscles, scapulothoracic winging, and decreased strength of the abdominal wall. 12 Scoliosis ${ }^{13}$ and decreased bone density ${ }^{14}$ are frequently diagnosed in children with PD. The cardiac muscle is frequently affected in all forms of PD and cases of serious
Correspondence: Bruno Corrado, Department of Public Health, University of Naples "Federico II", Via S. Pansini 5, 80131 Naples, Italy.

Tel.: +39.081.7462796 - Fax: +39.081.7462881. E-mail: bruno.corrado@unina.it

Key words: Pompe Disease; Glycogen Storage Disease Type II; Myopathies; Rehabilitation; Physical Therapy Modalities.

Contributions: B.C. and G.C. contributed to the conception and design of the study, to the acquisition and interpretation of data, and to the writing of the manuscript. C.S.I. participated in revising critically the article. B.C., G.C. and C.S.I. gave final approval of the version to be submitted.

Conflict of interest: the authors declare no potential conflict of interest.

Funding: none.

Received for publication: 10 January 2019

Accepted for publication: 10 April 2019.

This work is licensed under a Creative Commons Attribution NonCommercial 4.0 License (CC BY-NC 4.0).

CCopyright B. Corrado et al., 2019

Licensee PAGEPress, Italy

Neurology International 2019; 11:7983

doi:10.4081/ni.2019.7983

arrhythmias or Wolff-Parkinson-White syndromes have been reported in the literature. 15 Recent studies have highlighted cases of pre-symptomatic hyperCKemia. ${ }^{16}$ Finally, several studies indicate that congenital molecular alterations in neuromuscular diseases, like PD, promote cognitive dysfunctions. ${ }^{17,18}$

In 2015, early diagnosis of the pediatric forms of $\mathrm{PD}$ was included in the Recommended Uniform Screening Panel (RUSP) by the national study commission on rare and hereditary neonatal disorders. ${ }^{19}$ In particular, the commission recommended an evaluation of GAA enzyme activity via a dried blood spot (DBS) test. In cases of insufficient or nonexistent activity, an analytical examination should be made of the systems involved in order to identify the genetic and pathological type. The same algorithm can be applied to the late-onset forms. With regard to treatment, until a few years ago PD was only treated via supportive care. A real turning point in the treatment of PD came in 2007, when enzyme replacement therapy (ERT) was approved for patients with infantile forms of PD. In 2010 ERT was also approved for the late- 
onset form. In ERT, an extract of recombinant human GAA (also partially extracted from animals) is administered intravenously. This enzyme breaks down the accumulation of glycogen, particularly on the cardiac muscle. ${ }^{20}$ ERT has led to a clear decrease in short-term mortality and an improvement in respiratory and cardiac disorders in patients with PD. 20-24 In particular, significant increases have been reported in the time spent by patients without a ventilator, thereby allowing for greater autonomy and the achievement of new motor abilities. 24,25 Simultaneous with the benefits of managing vital functions in PD, the change in phenotypic expression introduced by ERT has led to the appearance of additional clinical problems related primarily to rehabilitation. The clinical picture of these patients (with either IOPD or LOPD) is characterized by muscular weakness, with the appearance of facial muscle asthenia, ptosis, insufficiency of the hip flexors and ankle dorsiflexors, difficulty walking, posture disorders, and scoliosis. ${ }^{13,26}$

Based on the above, it is clear that the need for a rehabilitative framework for patients with PD represents a gold standard in managing this syndrome. To date, different authors have developed general guidelines for physiotherapy programs for PD. Clinton et al., ${ }^{27}$ for example, recommend team management that integrates interventions by cardiologists, pulmonologists, physiatrists, physiotherapists and occupational therapists (Table 1 shows all the interventions proposed). Despite this, clinical evidence for the rehabilitative management of this syndrome is rare. The scope of the present review is to look for better available EBM regarding rehabilitation in patients with PD in order to establish practical recommendations for clinical conduct.

\section{Materials and Methods}

A systematic review of the literature was made using the following databases: Pubmed, Pedro, Cinhal, Cochrane Library, EDS Base Index, and Trip. The databases were accessed in October 2018. The PICO method was used to formulate the clinical query according to the following parameters: 28

- Population: PD patients

- Intervention: physiotherapy

- Comparison: inactive control groups

- Outcome: respiratory function improvements, improvements in range of motion/muscular strength

\section{Inclusion criteria and keywords}

Articles considered valid for the review addressed randomized controlled trials (RCT) or cohort studies relating to rehabilitation in patients with $\mathrm{PD}$, with no time limit, but with a study sample of at least six subjects. Only articles in English were selected. Table 2 summarizes the keywords used to perform the search.

\section{Results}

A total of 1665 results emerged from the search. Figure 1 shows the process to progressively select the articles found. With an initial screening, all results reported multiple times in the database (437 articles) were eliminated. Following this, a secondary screening of the title and abstract was made, resulting in the elimination of (a) article not dealing with the rehabilitation in patients with PD and (b) types of publications different from what was stated in the inclusion criteria (conference proceedings, extracts from textbooks, single expert opinions, animal-based studies). This second screening allowed an additional 1210 results to be eliminated. Therefore, the fulltext version of a total of 18 articles was assessed. These results were divided thus: 4 studies on individual cohorts, 3 case series, 5 case reports, 3 exploratory studies, and 3 literature reviews addressing the diagnostic procedure for PD.

The 4 studies in the first group were therefore admitted to the final phase of the review. From the detailed analysis of these articles, two approaches to rehabilitation in patients with PD were identified: respiratory muscle rehabilitation and physical activity. The main data used in all the studies included are reported in Table 3.29-32

\section{Re-education of the respiratory muscles}

A total of 3 studies ${ }^{29-31}$ dealt with the management of respiratory muscles in patients with PD. Chest physical therapy was always practiced as an adjuvant therapy to ERT. All the studies examined were single cohorts. The population studied by

Table 1. Evaluation and rehabilitation of PD according to the recommendations by Clinton et al.27

\section{Clinical recommendations}

Preliminary assessment with a pulmonologist or cardiologist before performing aerobic exercises or physiotherapy

Evaluate 6MWT at time 0

Include in rehabilitation programs walking, treadmill, use of the cyclette, the hydrokinetic therapy, swimming, muscle strengthening

Avoid excessively tiring exercises; avoid immobility

Give emphasis to aerobic exercises

Instruct the patient to monitor heart rate and breathing during exercise

Use preventive braces for contractures while the patient is in a wheelchair, and adequate supports when standing

Evaluate corrective surgery for scoliosis above $30-40^{\circ}$

Table 2. Keyword used for the search.

\begin{tabular}{lcc}
\hline Keyword 1 & Keyword 2 & Boolean operator \\
Pompe Disease & Physiotherapy & AND/OR \\
Pompe Disease & Rehabilitation & AND/OR \\
\hline Pompe Disease & Management & AND/OR \\
Glycogen Storage Disease Type 2 & Physiotherapy & AND/OR \\
\hline Glycogen Storage Disease Type 2 & Rehabilitation & AND/OR \\
Glycogen Storage Disease Type 2 & Management & AND/OR \\
\hline
\end{tabular}


Jevnikar et al.,29 and Aslan et al.,31 was composed of patients with LOPD, while only the article by $\mathrm{Smith}^{30}$ regarded a population with IOPD, which was already severely compromised from the respiratory point of view (patients ventilated for multiple hours per day). With regard to the intervention proposed, Jevnikar ${ }^{29}$ followed a protocol of inspiratory muscle training against fixed resistance (threshold). In particular, the patient was first studied with spirometry and then coached to perform 45 minutes of daily training: 15 minutes reaching $30 \%$ of the maximal inspiratory pressures (MIP) registered and the remaining time in deep breathing. Smith, ${ }^{30}$ instead, developed a protocol in which patients with IOPD followed inspiratory training three times per week in preparation for surgery for diaphragm gene therapy. Using modified equipment (Threshold PEP or AccuPEEP), patients followed 3-4 series of 6-10 active inhalations during which the maximum tolerated volume should be reached while maintaining $\mathrm{SpO}_{2}>92 \%$. Finally, Aslan developed an inspiratory muscle training program with a threshold lasting 8 weeks in which the 8 patients recruited were asked to perform the exercise two times per day. ${ }^{31}$ The level of required inspiratory pressure started at $30 \%$ of MIP registered with spirometry, increasing by $2 \mathrm{~cm}$ $\mathrm{H}_{2} \mathrm{O}$ each week.

With regard to the outcomes recorded, the levels of MIP were used in all studies, ${ }^{29-}$ 31 while only the study by $\mathrm{Smith}^{30}$ examined volume, flux, and post-inhalation compensation time with a threshold (at some points excluding the mechanical ventilation in the children studied). In addition, the electromyographic response of the diaphragm after training was evaluated. Lastly, the study by Aslan ${ }^{31}$ assessed the values of peak cough flow (PCF), forced vital capacity (FVC), the $\mathrm{FVC} /$ forced expiratory volume ratio in 1 second (FEV1), quality of life using the Nottingham Health Profile (NHP), and quality of sleep using the Pittsburgh Sleep Quality Index (PSQI). The follow-up period varied among the different studies: up to 24 months in the trial by Jevnikar, ${ }^{29}$ one year in the study by Smith, ${ }^{30}$ and 24 weeks in the study by Aslan. ${ }^{31}$

Finally, with respect to the results obtained, the study by Jevnikar ${ }^{29}$ highlighted a significant increase in MIP values, in agreement with what was demonstrated by Aslan. ${ }^{31}$ This data appears to contrast with what emerged from the study by Smith, 30 in which the pressure values did not change after the intervention. However, these values refer to a pediatric population in part already fully dependent on mechanical ven-

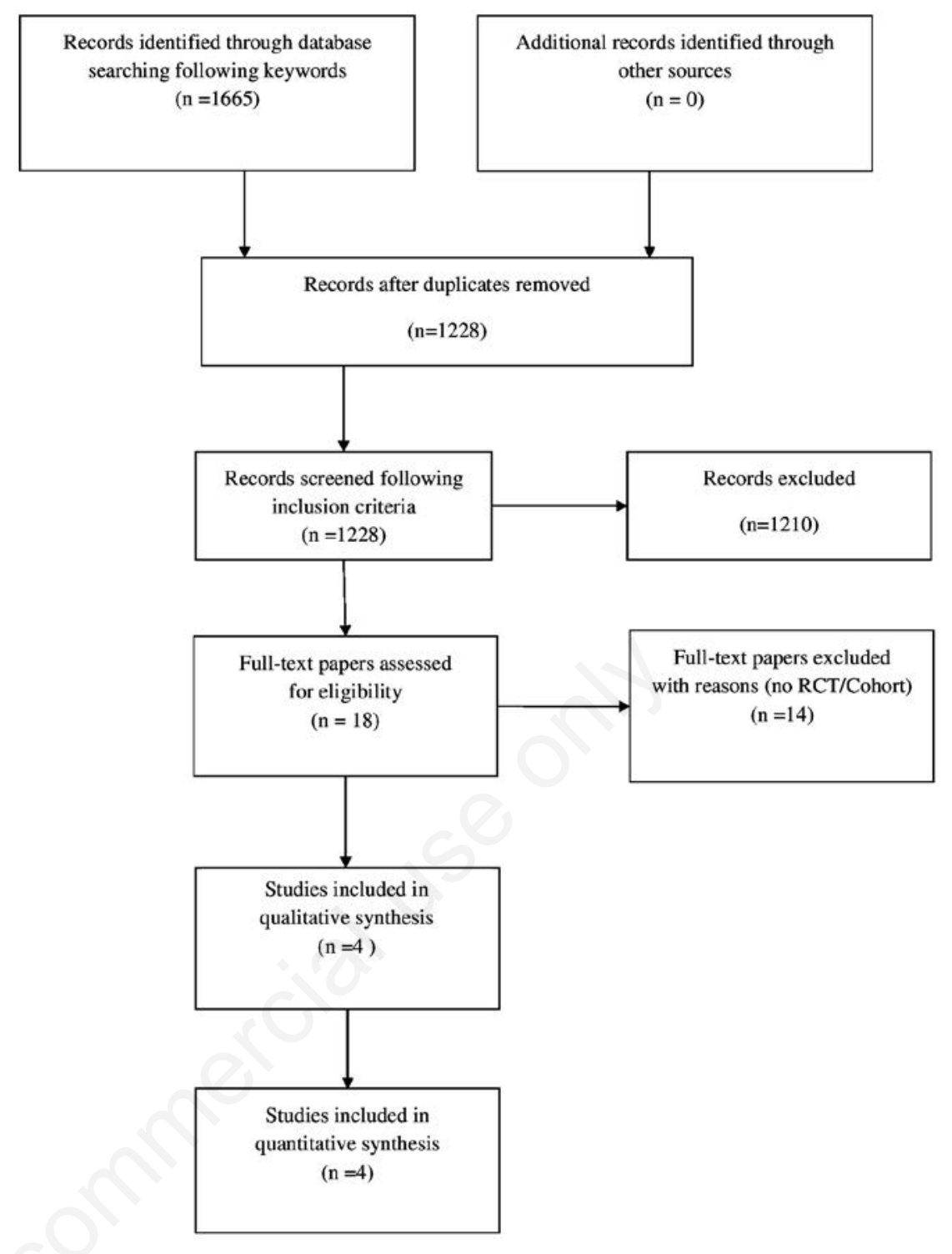

Figure 1. Selection of articles for the review.

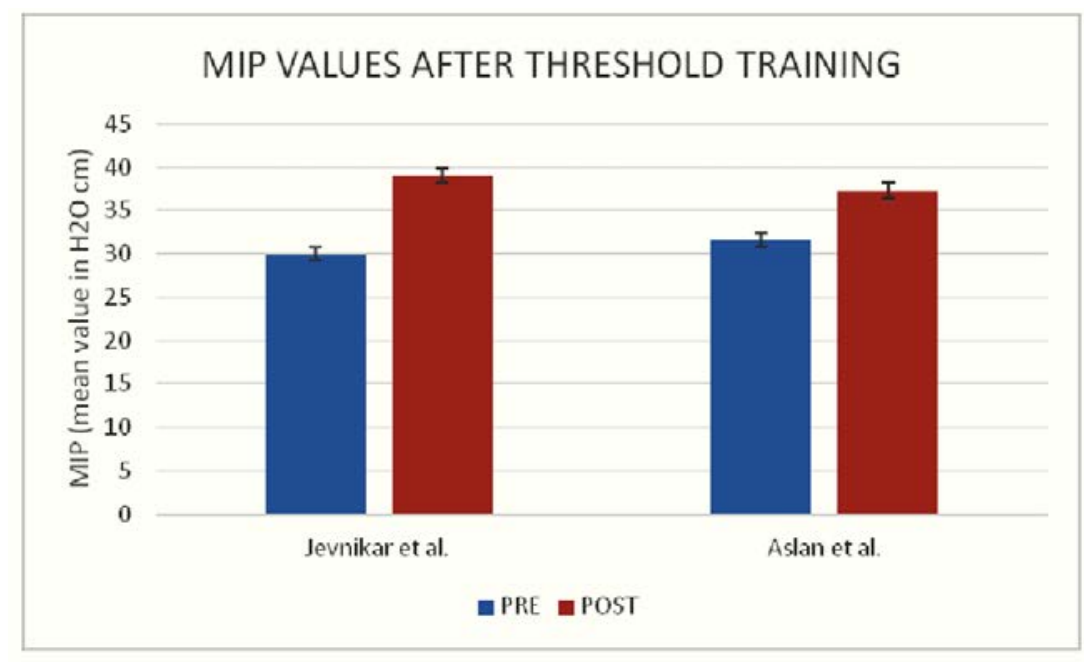

Figure 2. MIP values after threshold training according to Jevnikar ${ }^{29}$ and Aslan ${ }^{31}$ studies. 
tilation. With regard to the indicators of success, Smith ${ }^{30}$ found an increase in the compensatory response to threshold at only 180 days, with an increase in maximum voluntary ventilation in subjects and evidence of diaphragm activity on the electromyography. Finally, Aslan ${ }^{31}$ reported no modification in quality of sleep or quality of life at the end of the proposed protocol. Figure 2 shows a comparison of MIP values between Aslan $^{31}$ and Jevnikar. ${ }^{29}$

\section{Aerobic exercise and general physi- cal activity}

Only one article addressed protocols for general physical exercise in patients with PD. 32 The study, already presented previously at the sixth European innovation summit on $\mathrm{PD}, 33$ was conducted on a population of 25 patients with LOPD (23 completed the study), all of whom were undergoing ERT. The rehabilitation program, which lasted 12 weeks, included 36 sessions of aerobic training using a cycle ergometer, active resistance exercises (weights/mat activities), and core stability training. The authors evaluated the following as outcome measures: fatigue (using the Fatigue Severity Scale, FSS), plasma values of creatine kinase (CK; every two weeks), oxygen consumption during exercise (maximal oxygen uptake peak - $\mathrm{VO}_{2}$ peak - and max- imum work capacity - WMAX), maximum ventilatory threshold (VT), tolerance to exertion (using the Borg scale and six minute test), duration of hold in core stability exercises, muscular function (using the quick motor function test), and body composition (using dual X-ray absorptiometry, DXA). The authors report statistically significant increases in muscular endurance indices $\left(\mathrm{VO}_{2}\right.$ peak, WMAX, and VT), an increase in the duration of hold in core stability exercises, and the muscular strength of some groups (hip flexors, shoulder abductors). All the other outcomes evaluated were stable.

\section{Discussion}

The present review complies with the standards defined by the PRISMA statement for methodological quality of a literature review. 34 From what was illustrated above, rehabilitation in the management of patients with PD is a useful tool to strengthen the effects of ERT, contributing to the patient's greater respiratory and muscular compliance. In fact, in all studies examined, the two treatments were made simultaneously.29-32 However, this topic has been undervalued by the scientific literature. Indeed, from our review, no RCT or cohort studies with controlled groups were found. The studies that were found were only experiments with single cohorts, with a very small sample (8-9 subjects for the respiratory rehabilitation studies, 23 in the only motor re-education study). This obviously represents the main limitation and source of bias of the present review, and in general, of studies on rehabilitation in patients with rare neuromuscular pathologies. $35-39$ With regard to indications deriving from our review, rehabilitation methods aimed at reinforcing the respiratory muscles seem to increase MIP levels with a statistically significant difference, ${ }^{29,31}$ while only one publication ${ }^{30}$ deviates from this trend. It should be mentioned that the study in question regarded a notably different population of patients with respect to both age of onset (IOPD) and ventilatory impairment (5 patients already depended on mechanical ventilation for most of the day). The main changes observed in this case were reported in the follow-up at 180 days (increase in flows and volumes of inspiratory rehabilitation after training). No other substantial changes were described with respect to the respiratory profile of the participants. The validity of inspiratory training with a threshold with level III evidence, represents the greatest innovation in our study. The guidelines currently drafted for PD advise other therapeutic measures (management of

Table 3. Summary of included studies.

\begin{tabular}{|c|c|c|c|c|}
\hline Study & Design & Participants & Intervention & Outcome measures \\
\hline Jevnikar et al. ${ }^{29} 2015$ & Single Cohort & $n=8$ & $\begin{array}{l}\text { Exp }=\text { ERT +respiratory } \\
45 \mathrm{~min} / \text { day } \times 24 \text { months }\end{array}$ & $\begin{array}{l}\text { - } \text { MIP, MEP } \\
\text { muscle training with threshold } \\
\text { - Follow up: } 0,3,6,9,12,24 \text { month }\end{array}$ \\
\hline Smith et al. ${ }^{30} 2017$ & Single Cohort & $n=9$ & $\begin{array}{l}\text { Exp }=\text { ERT }+ \text { respiratory muscle } \\
\text { training with PEEP } \\
6 / 10 \text { active breaths } \times 4 \text { /day } \times 3 \text { months }\end{array}$ & $\begin{array}{l}\text { - Inspired tidal volume (VT), } \\
\text { peak inspiratory flow (PIF), } \\
\text { inspiratory pressure (PI), } \\
\text { inspiratory time (TI), expiratory time (TE) } \\
\text { - Borg scale } \\
\text { - MIP } \\
\text { - Volume, flow and timing compensatory responses to } \\
\text { inspiratory loads were evaluated using Threshold } \\
\text { training devices } \\
\text { - EMG } \\
\text { - Follow up }=0,90,180,365 \text { day }\end{array}$ \\
\hline Aslan et al.31 2016 & Single Cohort & $n=9$ & $\begin{array}{l}\text { Exp }=\text { ERT }+ \text { respiratory muscle training } \\
\text { with threshold } \\
15 \text { min } \times 2 \text { /day } \times 8 \text { wk }\end{array}$ & $\begin{array}{ll}\text { - } & \text { MIP, Cough peak flow(PCF) } \\
\text { - } & \text { Forced vital capacity (FVC), FVC/FEV1 } \\
\text { - } & \text { HRQoL (Nottingham Health Profile) } \\
\text { - } & \text { Sleep quality (Pittsburgh Sleep Quality Index -PSQI). } \\
\text { - } & \text { Follow up }=0,8,16,24 \mathrm{wk}\end{array}$ \\
\hline $\begin{array}{l}\text { Van der Berg } \\
\text { et al.32 } 2015\end{array}$ & Single Cohort & $\mathrm{n}=23$ & $\begin{array}{l}\text { Exp }=\text { standardized aerobic, resistance and } \\
\text { core stability exercises } 3 / w k \times 12 \text { wk }\end{array}$ & $\begin{array}{l}\text { - Fatigue severity scale } \\
\text { - Serum CK } \\
\text { - VO2 max and WMAX) } \\
\text { - Ventilator threshold (VT), } \\
\text { - Borg scale and 6MWT } \\
\text { - Resistance during core stability exercises, } \\
\text { - Quick motor function test } \\
\text { - } \text { - Follow up }=0,12,24 \mathrm{wk}\end{array}$ \\
\hline
\end{tabular}


bronchial secretions via drainage techniques, assistance coughing with an inexsufflator, continuous monitoring of sleep problems with the C-pap machine).27,40 However, the need remains for new RCTs with definitive EBM confirmation of these pulmonary rehabilitation protocols. Finally, with regard to the effectiveness of rehabilitative treatment based on aerobic and resistance training, the data uncovered refer only to a study by van Der Berg et al. 33 The trend of the sample studied shows a positive effect on the strength and endurance of some muscle groups, as well as on strength parameters of the core stability muscles. This seems to be confirmed in part by previous literature. Indeed, Kishnani et al. ${ }^{40}$ advise starting patients on mainly aerobic rehabilitative activities immediately after the general medical conditions are stabilized, avoiding activities that excessively stimulate the weakened muscle groups in eccentric contractions. About this topic as well, however, it is necessary to make a deeper scientific investigation by carrying out studies with greater evidence (RCTs) and a larger sample.

\section{Conclusions}

The present systematic literature review highlights that no sufficient studies on the methodological level exist today to support rehabilitation in the management of patients with PD. The cohort studies that are available suggest the use of inspiratory muscle training with a threshold to increase average MIP values (level III evidence) and rehabilitation programs based on physical aerobic exercise. However, new, broader studies are needed to definitively validate the abovementioned recommendations.

\section{References}

1. Mellies U, Lofaso F. Pompe disease: a neuromuscular disease with respiratory muscle involvement. Respir Med 2009;103:477-84.

2. Hirschhorn R, Reuser AJ. Glycogen storage disease type II: acid alpha-glucosidase (acid maltase) deficiency. In: Scriver CR, Beaudet A, Sly WS, Valle $\mathrm{D}$, eds. The metabolic and molecular bases of inherited disease. 8th ed. New York: McGraw-Hill; 2001. pp 3389420.

3. Ausems MG, Verbiest J, Hermans MP et al. Frequency of glycogen storage disease type II in The Netherlands: implications for diagnosis and genetic counselling. Eur J Hum Genet 1999;7: 713-6.

4. Pittis MG, Donnarumma M, Montalvo $\mathrm{AL}$, et al. Molecular and functional characterization of eight novel GAA mutations in Italian infants with Pompe disease. Hum Mutat 2008;29:E27-36.

5. Gort L, Coll MJ, Chabás A. Glycogen storage disease type II in Spanish patients: high frequency of c.10761G\&gt; C mutation. Mol Genet Metab 2007;92:183-7.

6. Dasouki M, Jawdat O, Almadhoun O, et al. Pompe disease: literature review and case series. Neurol Clin 2014;32:75176 .

7. Shintani T, Klionsky DJ. Autophagy in health and disease: a double-edged sword. Science 2004;306:990-5.

8. Huang J, Klionsky DJ. Autophagy and human disease. Cell Cycle 2007;6: 1837-49.

9. Mizushima N, Levine B, Cuervo AM, et al. Autophagy fights disease through cellular self-digestion. Nature 2008;451:1069-75.

10. Nixon RA. The role of autophagy in neurodegenerative disease. Nat Med 2013;19:983-97.

11. van der Beek NA, de Vries JM, Hagemans ML, et al. Clinical features and predictors for disease natural progression in adults with Pompe disease: a nationwide prospective observational study. Orphanet J Rare Dis 2012;7:88

12. MENA Pompe Working Group, Al Jasmi F, Al Jumah M, et al. Diagnosis and treatment of late-onset Pompe disease in the Middle East and North Africa region: consensus recommendations from an expert group. BMC Neurol 2015;15:205.

13. Roberts M, Kishnani PS, van der Ploeg AT, et al. The prevalence and impact of scoliosis in Pompe disease: lessons learned from the Pompe registry. Mol Genet Metab 2011;104:574-82.

14. van den Berg LE, Zandbergen AA, van Capelle CI, et al. Low bone mass in Pompe disease: muscular strength as a predictor of bone mineral density. Bone 2010;47:643-9.

15. Müller-Felber W, Horvath R, Gempel $\mathrm{K}$, et al. Late onset Pompe disease: clinical and neurophysiological spectrum of 38 patients including long-term followup in 18 patients. Neuromuscul Disord 2007;17:698-706.

16. Lukacs Z, Nieves Cobos P, Wenninger $\mathrm{S}$, et al. Prevalence of Pompe disease in 3,076 patients with hyperCKemia and limb-girdle muscular weakness. Neurology 2016;87:295-8.

17. Orsini M, Carolina A, Ferreira AF, et al.
Cognitive impairment in neuromuscular diseases: A systematic review. Neurol Int 2018;10:7473.

18. Ebbink BJ, Poelman E, Plug I, Lequin $\mathrm{MH}$, et al. Cognitive decline in classic infantile Pompe disease: An underacknowledged challenge. Neurology 2016;86:1260-1.

19. American College of Medical Genetics Newborn Screening Expert Group. Newborn screening: towards a uniform screening panel and system. Pediatrics 2006;117:S296-307.

20. Amalfitano A, Bengur AR, Morse RP, et al. Recombinant human acid alphaglucosidase enzyme therapy for infantile glycogen storage disease type II: results of a phase I/II clinical trial. Genet Med 2001;3:132-8.

21. Kishnani PS, Nicolino M, Voit T, et al. Chinese hamster ovary cell-derived recombinant human acid alpha-glucosidase in infantile-onset Pompe disease. J Pediatr 2006;149:89-97.

22. Klinge L, Straub V, Neudorf U, Voit T. Enzyme replacement therapy in classical infantile pompe disease: results of a ten month follow-up study. Neuropediatrics 2005;36:6-11.

23. Klinge L, Straub V, Neudorf U, et al. Safety and efficacy of recombinant acid alpha-glucosidase (rhGAA) in patients with classical infantile Pompe disease: results of a phase II clinical trial. Neuromuscul Disord 2005;15:24-31.

24. Nicolino M, Byrne B, Wraith JE, et al. Clinical outcomes after long-term treatment with alglucosidase alfa in infants and children with advanced Pompe disease. Genet Med 2009;11:210-9.

25. Gungor D, Kruijshaar ME, Plug I, et al. Impact of enzyme replacement therapy on survival in adults with Pompe disease: results from a prospective international observational study, Orphanet J Rare Dis 2013;8:49.

26. Case LE, Kishnani PS. Physical therapy management of Pompe disease. Genet Med 2006;8:318-27.

27. Clinton LJ, Nascimento OJ, Oliveira AS, et al. Guidelines for the diagnosis, treatment and clinical monitoring of patients with juvenile and adult Pompe disease. Arq Neuropsiquiatr 2016;74:166-76.

28. Higgins JPT, Green S. Cochrane Handbook for Systematic Reviews of Interventions, Version 5.1.0. The Cochrane Collaboration. 2013

29. Jevnikar M, Kodric M, Cantarutti F, et al. Respiratory muscle training with enzyme replacement therapy improves muscle strength in late-onset Pompe disease. Mol Genet Metab Rep 
2015;5:67-71.

30. Smith BK, Martin AD, Lawson LA, et al. Inspiratory muscle conditioning exercise and diaphragm gene therapy in Pompe disease: Clinical evidence of respiratory plasticity. Exp Neurol 2017;287:216-24.

31. Aslan GK, Huseyinsinoglu BE, Oflazer $\mathrm{P}$, et al. Inspiratory muscle training in late-onset Pompe disease: the effects on pulmonary function tests, quality of life, and sleep quality. Lung 2016;194:55561 .

32. van den Berg L, Favejee MM, Wens SC, et al. Safety and efficacy of exercise training in adults with Pompe disease: evaluation of endurance, muscle strength and core stability before and after a 12 week training program. Orphanet J Rare Dis 2015;10:87.
33. van den Berg L, Favejee MM, Wens SC, et al. Effects of exercise training in 23 adults with Pompe disease receiving enzyme therapy. BMC Musculoskelet Disord 2013;14:17.

34. Moher D, Liberati A, Tetzlaff J, Altman DG; PRISMA Group. Preferred Reporting Items for Systematic Reviews and Meta-Analyses: The PRISMA Statement. PLoS Med 2009;6:e1000097.

35. Corrado B, Ciardi G. Facioscapulohumeral distrophy and physiotherapy: a literary review. J Phys Ther Sci 2015;27:2381-5.

36. Corrado B, Ciardi G, Bargigli C. Rehabilitation management of the Charcot-Marie-Tooth syndrome: a systematic review of the literature. Medicine (Baltimore) 2016;95:e3278
37. Corrado B, Ciardi G. Hypermobile Ehlers-Danlos syndrome and rehabilitation: taking stock of evidence based medicine: a systematic review of the literature. J Phys Ther Sci 2018;30:843-7.

38. Schreuder L, Peters G, Nijhuis-van der Sanden R, Morava E. Aerobic exercise in children with oxidative phosphorylation defects. Neurol Int 2010;2:e4.

39. Kataoka H, Nanaura H, Kinugawa K, et al. Risk of unsuccessful noninvasive ventilation for acute respiratory failure in heterogeneous neuromuscular diseases: a retrospective study. Neurol Int 2017;9:6904.

40. Kishnani PS, Steiner RD, Bali D, et al. Pompe disease diagnosis and management guideline. Genet Med 2006;8:26788 . 